\title{
High-resolution 3D scanning X-ray microscopes at the Swiss Light Source
}

Mirko Holler*, Jörg Raabe, Ana Diaz, Manuel Guizar-Sicairos, Esther H. R. Tsai, Michal Odstrcil, Andreas Menzel, Oliver Bunk

Paul Scherrer Institut, 5232 Villigen PSI, Switzerland

*mirko.holler@psi.ch

Ptychography is a promising method to overcome limitations of traditional X-ray imaging schemes in resolution and depth-of-field. A sample is scanned at overlapping positions through a coherent beam of X-rays and for each spot a far-field diffraction pattern is recorded. A real-space image is reconstructed using iterative algorithms. At multi-keV photon energies the penetration depth allows imaging thick samples where 3D reconstructions are crucial for understanding the internal structure of the sample measured. This can be achieved by measuring the sample at various orientations and combining these measurements via computed tomography.

As a scanning technique ptychography remains sensitive to inaccuracies in sample positioning, which deteriorates the resolution and signal-to-noise ratio. Additionally, many sample systems suffer from radiation induced changes that alter the sample's structure during the measurement, which can be alleviated by cryogenic sample conditions. The latter is also essential for the measurement of cryogenically fixed biological samples in a near to native state.

In 2010 the OMNY (tOMography Nano $\mathrm{crYo}^{1}{ }^{1}$ project started at the Paul Scherrer Institut with the goal to develop a dedicated instrument to perform tomography at the nano-scale on biological samples and condensed matter physics samples using ptychography in a controlled sample environment.

To achieve high position accuracy, dedicated laser interferometry ${ }^{2,3}$ was developed which is compatible with scanning (for ptychography) and rotation (for tomography). This metrology measures the relative position between sample and a Fresnel zone plate, which is used to define the illumination on the sample. This scheme is now implemented in two instruments, one called flOMNI (flexible tOMography Nano Imaging) ${ }^{4}$ for measurements at room temperature and atmospheric pressure, and OMNY for measurements in ultra-high vacuum at cryogenic conditions.

In the presentation both instruments will be explained in detail. Both use the same customdeveloped sample holder, the OMNY pin $^{5}$, which has proven to work well at both, roomtemperature and cryogenic conditions. The setups are operated at the cSAXS beamline. We will show the routinely used procedures for setting up and operating the instruments. Latest improvements but also current and future limitations will be discussed.

While flOMNI was initially intended as feasibility study for the cryogenic instrument, it became clear that limitations created by an in-vacuum cryo instrument are unfavorable for many experiments. flOMNI is much more easily adapted to non-standard measurement geometries, for example for in-situ experiments, and it is also simpler to set up and operate. Therefore flOMNI remains in regular use for measuring sample systems that are insensitive to radiation and the setup is continuously improved. 
In high contrast samples, such as integrated circuits, a 3D resolution of $14.6 \mathrm{~nm}$ was demonstrated in a $10 \mu \mathrm{m}$ diameter sample extracted from an Intel $\mathrm{CPU}^{6}$. Another example demonstrating the routine operation and thus throughput of the setup is the visualization of a complete redox cycle in a solid oxide cell (SOC) electrode, which required several measurements of the same sample after several ex-situ treating steps ${ }^{7}$. An example of the flexibility of the setup is its modification for tomography with magnetic contrast ${ }^{8}$. Here X-ray magnetic dichroism is used to obtain magnetic contrast. The magnetization parallel to the axis of rotation cannot be determined. Therefore, a dual-axis tomography had to be realized and two measurements of the same sample at different orientations were combined in the data analysis.

The design of $\mathrm{OMNY}^{1}$ started in 2012 and the concepts were presented at the XRM conference 2014 in Melbourne. In 2015 OMNY was in operation for the first time and at liquid nitrogen temperature. Already then it demonstrated sub-30 nm 3D imaging on a highcontrast biological sample, a beetle wing scale ${ }^{9}$. Other examples include the measurement of polymer structures ${ }^{10}$ and the imaging of unstained and cryogenically fixed tissue ${ }^{11}$. With a few years of experience with OMNY, limitations and current difficulties will be discussed.

The two instruments are currently complemented by a third one measuring in the laminography geometry. Here the axis of rotation is not perpendicular to the x-ray beam which makes the setup better suited for flat samples, such as integrated circuits. The setup is not compatible with the interferometry scheme used in flOMNI and OMNY and thus a different strategy is used here. The concept and status of the LAMNI (lAMinography Nano Imaging) will be briefly presented.
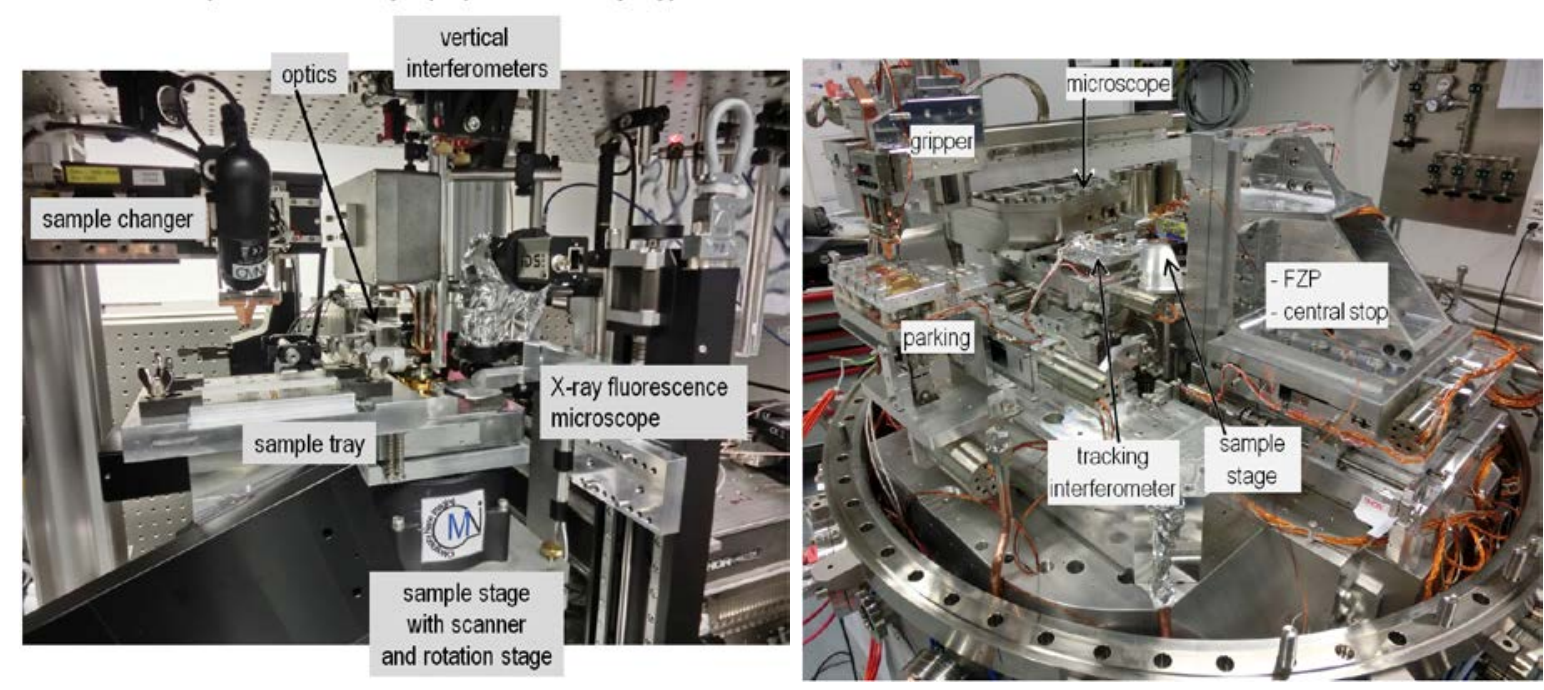

Figure. 1 (left) Photograph of the flOMNI instrument. (right) Photograph of the open vacuum vessel of OMNY.

\section{Acknowledgments}

We thank the Swiss National Science Foundation SNF for the support of the development of the OMNY instrument (R'EQUIP, no. 145056, "OMNY") and the Competence Centre for Materials Science and Technology (CCMX) of the ETH-Board, Switzerland E. H. R. Tsai is supported by the Swiss National Science Foundation (SNSF) grant numbers 200021_152554 and 200020_169623. 
[1] Holler M, Raabe J, Diaz A, Guizar-Sicairos M, Wepf R, Odstrcil M, et al. OMNY - a tOMography Nano crYo stage. Rev Sci Instrum 2018.

[2] Holler M, Raabe J. Tracking type laser interferometer for objects with rotational degrees of freedom. Patent application WO 2012079875 A1 2010.

[3] Holler M, Raabe J. Error motion compensating tracking interferometer for the position measurement of objects with rotational degree of freedom. Optical Engineering 54, no. 5 (2015): 05410.

[4] Holler M, Raabe J, Diaz A, Guizar-Sicairos M, Quitmann C, Menzel A, et al. An instrument for 3D x-ray nano-imaging. Review of Scientific Instruments 2012, 83(7): 073703.

[5] Holler M, Raabe J, Wepf R, Shahmoradian SH, Diaz A, Sarafimov B, et al. OMNY PIN-A versatile sample holder for tomographic measurements at room and cryogenic temperatures. Review of Scientific Instruments 2017, 88(11): 113701.

[6] Holler M, Guizar-Sicairos M, Tsai EHR, Dinapoli R, Muller E, Bunk O, et al. Highresolution non-destructive three-dimensional imaging of integrated circuits. Nature 2017, 543(7645): 402.

[7] De Angelis S, Jørgensen PS, Esposito V, Tsai EHR, Holler M, Kreka K, et al. Ex-situ tracking solid oxide cell electrode microstructural evolution in a redox cycle by high resolution ptychographic nanotomography. Journal of Power Sources 2017, 360: 520527.

[8] Donnelly C, Guizar-Sicairos M, Scagnoli V, Gliga S, Holler M, Raabe J, et al. Threedimensional magnetization structures revealed with X-ray vector nanotomography. Nature 2017, 547(7663): 328-+.

[9] Wilts BD, Sheng X, Holler M, Diaz A, Guizar-Sicairos M, Raabe J, et al. Evolutionary-Optimized Photonic Network Structure in White Beetle Wing Scales. Advanced Materials 2017: 1702057-n/a.

[10] Pallon LKH, Nilsson F, Yu S, Liu DM, Diaz A, Holler M, et al. Three-Dimensional Nanometer Features of Direct Current Electrical Trees in Low-Density Polyethylene. Nano Lett 2017, 17(3): 1402-1408.

[11] Shahmoradian S, Tsai E, Diaz A, Guizar-Sicairos M, Spcyher L, Raabe J, et al. ThreeDimensional Imaging of Biological Tissue by Cryo X-Ray Ptychography. Sci Rep 7, no. 1 (2017): 6291. 
https://doi.org/10.1017/S1431927618013223 Published online by Cambridge University Press 